\title{
ON THE EXISTENCE OF SMOOTH SOLUTIONS IN ONE-DIMENSIONAL NONLINEAR THERMOELASTICITY WITH SECOND SOUND
}

\author{
BY \\ MICHAEL A. TARABEK \\ Southern Illinois University, Carbondale, Illinois
}

1. Introduction. In the absence of dissipation, smooth motions of nonlinear elastic materials will generally break down due to the formation of shocks (see, e.g., Lax [15], MacCamy and Mizel [16]). It is interesting to consider situations in which the destabilizing effects of a nonlinear elastic response compete with a dissipative mechanism. An important example of such a dissipative mechanism in an elastic material is the conduction of heat.

In classical one-dimensional thermoelasticity, heat conduction is assumed to be governed by Fourier's law. Although Fourier's law provides a description of heat conduction that is useful under a wide range of conditions, experiments on certain dielectric crystals at very low temperatures have produced results that are inconsistent with those predicted by Fourier's law (cf. the references cited in $[2,14]$ ). Whereas classical theory predicts an infinite speed of propagation for thermal disturbances, these crystals transmit such disturbances as wavelike pulses, travelling at finite speeds. This phenomenon is known as second sound. One of the earliest attempts to model second sound was made by Cattaneo [1].

In this paper, we establish the global existence of a smooth solution to the equations of one-dimensional nonlinear thermoelasticity when Fourier's law is replaced by Cattaneo's law. The second law of thermodynamics is used to restrict the forms of the constitutive relations for stress and internal energy. The constitutive relations, the laws of momentum balance and energy balance, and some assumptions motivated by physical considerations yield a hyperbolic system of equations. Thus a finite speed of propagation is predicted.

Although the dissipative effects of heat conduction induced by Cattaneo's law are not as strong as those induced by Fourier's law, there exists a unique globally defined classical solution to the initial-value problem provided the initial data are smooth and lie sufficiently close to equilibrium. Further, the solution will tend to equilibrium as time goes to infinity. Both the case of an unbounded body and the case of a

Received December 17, 1990.

1991 Mathematics Subject Classification. Primary 35L70.

This research was conducted under the supervision of William J. Hrusa and partially supported by the U.S. Air Force under contracts AFOSR-85-0307 and AFOSR-88-0265.

(C) 1992 Brown University 
bounded body with pinned and insulated ends are treated. When the body occupies the entire real line, some relations associated with the second law of thermodynamics are exploited to make up for the lack of a Poincaré inequality.

Some results have already been obtained for a rigid body in which the conduction of heat is governed by Cattaneo's law. Coleman, Fabrizio, and Owen [4, 5] determined the forms that the constitutive relations must take in order to be consistent with the second law of thermodynamics. Coleman, Hrusa, and Owen [8] then established the existence of a global, smooth classical solution when smooth data that lie sufficiently close to equilibrium are prescribed.

It should be noted that Cattaneo's equation is not the only attempt to model second sound. Gurtin and Pipkin [11] develop a general theory of heat flow in rigid bodies with memory which predicts finite speed of propagation for thermal disturbances. Chen and Gurtin [3] extend the theory to include deformable media and present an example for which the heat flux relation reduces to a form of Cattaneo's law. Another approach, first advanced by Müller [17] is to introduce a generalized temperature function and a suitably generalized form of the second law of thermodynamics. This results in a hyperbolic heat transport equation without modification of the Fourier heat conduction law. The survey paper of Chandrasekharaiah [2] presents a discussion of these and other theoretical approaches to second sound as well as a discussion of experimental results. Joseph and Preziosi [14] present a detailed chronology of published works on the subject of heat propagation by waves.

The organization and exposition of this paper is similar to that of [12] in which classical heat conduction is investigated. It is important to note that we again make crucial use of the thermodynamic relations to obtain the energy estimate for lowest order terms, an estimate that cannot be obtained using a Poincare inequality when the body is unbounded. Although much of the analysis here is similar to that of [12], the results differ slightly in that less smoothness is required of the initial data for the Cattaneo problem.

2. Thermodynamics. We consider a homogeneous body that has unit reference density and occupies an interval $\mathscr{B} \subset \mathbb{R}$ in its reference configuration. We assume that the motion is that of one-dimensional extension. For such a body, the laws of balance of momentum, balance of energy, and growth of entropy take the forms:

$$
\begin{gathered}
u_{t t}=\sigma_{x}+b, \\
e_{t}+q_{x}=\sigma \varepsilon_{t}+r, \\
\eta_{t} \geq \frac{r}{\theta}-\left(\frac{q}{\theta}\right)_{x},
\end{gathered}
$$

where $u$ is the displacement, $\varepsilon=u_{x}$ is the strain, $\sigma$ is the stress, $b$ is the body force, $e$ is the internal energy, $r$ is the heat supply, $\eta$ is the entropy, $\theta$ is the (absolute) temperature, and $q$ is the heat flux. We assume that these fields depend smoothly on the material point $x \in B$ and the time $t \geq 0$. Furthermore, the strain and temperature are required to satisfy

$$
\varepsilon>-1, \quad \theta>0 .
$$


Here, a subscript is used to indicate a partial derivative.

Define the free energy $\psi$ through the relation

$$
\psi=e-\theta \eta,
$$

and put

$$
g=\theta_{x}
$$

for the temperature gradient. Then we may combine Eqs. (2.2) and (2.3) to produce the Clausius-Duhem inequality

$$
\psi_{t}+\eta \theta_{t}-\sigma \varepsilon_{t}+\frac{q g}{\theta} \leq 0 .
$$

The constitutive relations that describe a thermoelastic medium in which heat conduction is governed by Cattaneo's law are

$$
\begin{aligned}
\psi & =\hat{\psi}(\varepsilon, \theta, q), & \sigma & =\hat{\sigma}(\varepsilon, \theta, q), \\
\eta & =\hat{\eta}(\varepsilon, \theta, q), & e & =\hat{e}(\varepsilon, \theta, q),
\end{aligned}
$$

and

$$
\tau(\varepsilon, \theta) q_{t}+q=-\kappa(\varepsilon, \theta) g
$$

where $\hat{\psi}, \hat{\sigma}, \hat{\eta}, \hat{e}, \tau$, and $\kappa$ are smooth functions. We shall refer to Eq. (2.9) as Cattaneo's law. Here $\tau$ is the thermal relaxation time and $\kappa$ is the thermal conductivity.

On physical grounds, it is reasonable to assume that there exists a temperature $\bar{\theta}$ such that when $\varepsilon=q=0$ and $\theta=\bar{\theta}$, the specific heat, the elastic modulus, the thermal relaxation time, and the thermal conductivity are positive while the stresstemperature modulus is nonvanishing; i.e.,

$$
\begin{gathered}
\hat{e}_{\theta}(0, \bar{\theta}, 0)>0, \quad \hat{\sigma}_{\varepsilon}(0, \bar{\theta}, 0)>0, \\
\tau(0, \bar{\theta})>0, \quad \kappa(0, \bar{\theta})>0, \\
\hat{\sigma}_{\theta}(0, \bar{\theta}, 0) \neq 0 .
\end{gathered}
$$

Using techniques developed in Coleman and Noll [7] and Coleman and Mizel [6], one can show that necessary and sufficient conditions for the Clausius-Duhem inequality (2.7) to hold are given by

$$
\begin{aligned}
& \hat{\psi}(\varepsilon, \theta, q)=\psi^{0}(\varepsilon, \theta)+\frac{1}{2} \chi(\varepsilon, \theta) q^{2}, \\
& \hat{\sigma}(\varepsilon, \theta, q)=\hat{\psi}_{\varepsilon}(\varepsilon, \theta, q) \\
& \hat{\eta}(\varepsilon, \theta, q)=-\hat{\psi}_{\theta}(\varepsilon, \theta, q)
\end{aligned}
$$

and

$$
\kappa \geq 0
$$

where

$$
\chi(\varepsilon, \theta)=\frac{\tau(\varepsilon, \theta)}{\theta \kappa(\varepsilon, \theta)} .
$$

(An excellent introduction to the techniques involved here can be found in [9].)

It is not universally accepted that the heat flux should appear in the free energy as a quadratic as is indicated by the methods of Coleman et al. (See, e.g., [10] in 
which Cattaneo's law is assumed, yet the free energy is taken to be independent of the heat flux.) We will not address this question here. We do note, however, that our conclusions will hold with less restrictive assumptions about the dependence of $\psi$ on $q$.

It follows from Eqs. (2.5) and (2.11) that the internal energy is given by

$$
\hat{e}(\varepsilon, \theta, q)=\hat{\psi}(\varepsilon, \theta, q)-\theta \hat{\psi}_{\theta}(\varepsilon, \theta, q),
$$

from which it follows that

$$
\hat{e}_{\theta}=-\theta \hat{\psi}_{\theta \theta} \quad \text { and } \quad \frac{\hat{\sigma}-\hat{e}_{\varepsilon}}{\hat{\sigma}_{\theta}}=\theta .
$$

The sufficiency of Eqs. (2.11), (2.12) is clear since

$$
\psi_{t}+\eta \theta_{t}-\sigma \varepsilon_{t}=\chi q q_{t},
$$

which when combined with Eqs. (2.9) gives

$$
\psi_{t}+\eta \theta_{t}-\sigma \varepsilon_{t}+\frac{q g}{\theta}=\frac{-q^{2}}{\kappa \theta} \leq 0 .
$$

Another useful identity, which follows from the balance of energy equation (2.2) and (2.15), is

$$
\theta \eta_{t}=r-q_{x}-\chi q q_{t}
$$

3. Statement of results. For simplicity, we assume that the heat supply $r$ and the body force $b$ vanish identically. (See the remarks following the statement of Theorem 2.) We further assume that the heat flux is given by Eq. (2.9). We seek smooth solutions to the system of equations $(2.1),(2.2),(2.9)$ when the initial values for displacement, velocity, temperature, and heat flux are given. Thus we consider the initial value problem

$$
\begin{gathered}
u_{t t}=\hat{\sigma}_{\varepsilon}(\varepsilon, \theta, q) u_{x x}+\hat{\sigma}_{\theta}(\varepsilon, \theta, q) \theta_{x}+\hat{\sigma}_{q}(\varepsilon, \theta, q) q_{x}, \\
\hat{e}_{\theta}(\varepsilon, \theta, q) \theta_{t}=\left[\hat{\sigma}-\hat{e}_{\varepsilon}\right](\varepsilon, \theta, q) u_{x t}-q_{x}-\hat{e}_{q}(\varepsilon, \theta, q) q_{t}, \\
\tau(\varepsilon, \theta) q_{t}+q=-\kappa(\varepsilon, \theta) \theta_{x},
\end{gathered}
$$

$\forall x \in \mathscr{B}, t \geq 0$

$$
\begin{array}{ll}
u(x, 0)=u_{0}(x), & u_{t}(x, 0)=u_{1}(x), \\
\theta(x, 0)=\theta_{0}(x), & q(x, 0)=q_{0}(x),
\end{array}
$$

$\forall x \in \mathscr{B}$ where $u_{0}, u_{1}, \theta_{0}$, and $q_{0}$ are prescribed functions. In the statements and proofs of the theorems, differentiation is to be interpreted in the sense of distributions. $L^{2}(\mathscr{B})$ denotes the space of square-integrable functions on $\mathscr{B}$, and $H^{k}(\mathscr{B})$ denotes the Sobolev space of functions in $L^{2}(\mathscr{B})$ whose derivatives through order $k$ belong to $L^{2}(\mathscr{B})$. We use $C\left([0, T) ; L^{2}(\mathscr{B})\right), L^{2}\left([0, T) ; L^{2}(\mathscr{B})\right)$, and $L^{\infty}\left([0, T) ; L^{2}(\mathscr{B})\right)$ to denote, respectively, the space of continuous, squareintegrable, and essentially bounded functions on $[0, T)$ whose values lie in $L^{2}(\mathscr{B})$. 
We assume that the thermodynamic relations (2.11) hold and that there is a fixed temperature $\bar{\theta}>0$ such that the physical assumptions (2.10) hold; that is,

$$
\begin{aligned}
& \hat{\psi}(\varepsilon, \theta, q)=\psi^{0}(\varepsilon, \theta)+\frac{1}{2} \chi(\varepsilon, \theta) q^{2} \\
& \hat{\sigma}(\varepsilon, \theta, q)=\hat{\psi}_{\varepsilon}(\varepsilon, \theta, q), \\
& \hat{\eta}(\varepsilon, \theta, q)=-\hat{\psi}_{\theta}(\varepsilon, \theta, q) \\
& \hat{e}_{\theta}(0, \bar{\theta}, 0)>0, \quad \hat{\sigma}_{\varepsilon}(0, \bar{\theta}, 0)>0, \\
& \tau(0, \bar{\theta})>0, \quad \kappa(0, \bar{\theta})>0, \\
& \hat{\theta}_{\theta}(0, \bar{\theta}, 0) \neq 0 .
\end{aligned}
$$

If the initial data are smooth and close to the equilibrium state $(0, \bar{\theta}, 0)$ in the sense of Sobolev norms, then the initial value problem (3.1)-(3.4) has a unique and globally defined classical solution. The precise results follow.

TheOREM 1. Let $\mathscr{B}=\mathbb{R}$. Let $\bar{\theta} \in(0, \infty)$ be given. Suppose that $\psi^{0}, \tau$, and $\kappa$ are in $C^{4}((-1, \infty) \times(0, \infty))$ and that Eqs. (3.5), (3.6) hold. Then there is a number $\rho>0$ such that whenever $u_{0}, u_{1}, \theta_{0}$, and $q_{0}: \mathscr{B} \rightarrow \mathbb{R}$ satisfy

$$
u_{0}^{\prime}, u_{1}, \theta_{0}-\bar{\theta}, q_{0} \in H^{2}(\mathscr{B})
$$

and

$$
\left\|u_{0}^{\prime}\right\|_{H^{2}(\mathscr{B})}^{2}+\left\|u_{1}\right\|_{H^{2}(\mathscr{B})}^{2}+\left\|\theta_{0}-\bar{\theta}\right\|_{H^{2}(\mathscr{B})}^{2}+\left\|q_{0}\right\|_{H^{2}(\mathscr{B})}^{2}<\rho^{2},
$$

where a prime indicates differentiation with respect to the single variable, then the initial-value problem $(3.1)-(3.4)$ has a unique solution $(u, \theta, q)$ with

$$
\begin{gathered}
u_{x}, u_{t}, u_{x x}, u_{x t}, u_{t t}, u_{x x x}, u_{x x t}, u_{x t t}, u_{t t t}, \theta-\bar{\theta}, \theta_{x}, \\
\theta_{t}, \theta_{x x}, \theta_{x t}, \theta_{t t}, q, q_{x}, q_{t}, q_{x x}, q_{x t}, q_{t t} \\
\in C\left([0, \infty) ; L^{2}(\mathscr{B})\right) \cap L^{\infty}\left([0, \infty) ; L^{2}(\mathscr{B})\right), \\
u_{x x}, u_{x t}, u_{t t}, u_{x x x}, u_{x x t}, u_{x t t}, u_{t t t}, \theta_{x}, \theta_{t}, \theta_{x x}, \\
\quad \theta_{x t}, \theta_{t t}, q, q_{x}, q_{t}, q_{x x}, q_{x t}, q_{t t} \\
\quad \in L^{2}\left([0, \infty) ; L^{2}(\mathscr{B})\right), \\
u_{x}, u_{t}, u_{x x}, u_{x t}, u_{t t}, \theta-\bar{\theta}, \theta_{x}, \theta_{t}, q, q_{x}, q_{t} \rightarrow 0 \\
\text { uniformly on } \mathscr{B}, \text { as } t \rightarrow \infty, \\
u_{x x}, u_{x t}, u_{t t}, \theta_{x}, \theta_{t}, q, q_{x}, q_{t} \rightarrow 0 \text { in } L^{2}(\mathscr{B}), \text { as } t \rightarrow \infty,
\end{gathered}
$$

and

$$
u_{x}(x, t)>-1, \quad \theta(x, t)>0 \quad \forall x \in \mathscr{B}, t \geq 0 .
$$

We now turn our attention to an analogous problem on a bounded interval. Suppose that, in its reference configuration, the body occupies the interval $[0,1]$ and obeys the boundary conditions

$$
u(0, t)=u(1, t)=q(0, t)=q(1, t)=0 \quad \forall t \geq 0 .
$$


The corresponding existence result is

Theorem 2. Let $\mathscr{B}=[0,1]$. Let $\bar{\theta} \in(0, \infty)$ be given. Suppose that $\psi^{0}$, $\tau$, and $\kappa$ are in $C^{4}((-1, \infty) \times(0, \infty))$. Suppose further that Eqs. (3.5), (3.6) hold. Then there is a number $\rho>0$ such that whenever $u_{0}, u_{1}, \theta_{0}$, and $q_{0}: \mathscr{B} \rightarrow \mathbb{R}$ satisfy Eqs. (3.7), (3.8) and

$$
\begin{gathered}
u_{0}(0)=u_{0}(1)=u_{1}(0)=u_{1}(1)=q_{0}(0)=q_{0}(1)=0, \\
u_{0}^{\prime \prime}(0)=u_{0}^{\prime \prime}(1)=\theta_{0}^{\prime}(0)=\theta_{0}^{\prime}(1)=0,
\end{gathered}
$$

then the boundary-value problem (3.1)-(3.4), (3.14) has a unique solution $(u, \theta, q)$ with

$$
\begin{gathered}
u, u_{x}, u_{t}, u_{x x}, u_{x t}, u_{t t}, u_{x x x}, u_{x x t}, u_{x t t}, u_{t t t}, \\
\theta-\bar{\theta}, \theta_{x}, \theta_{t}, \theta_{x x}, \theta_{x t}, \theta_{t t}, q, q_{x}, q_{t}, q_{x x}, q_{x t}, q_{t t} \\
\in C\left([0, \infty) ; L^{2}(\mathscr{B})\right) \cap L^{\infty}\left([0, \infty) ; L^{2}(\mathscr{B})\right), \\
u, u_{x}, u_{t}, u_{x x}, u_{x t}, u_{t t}, u_{x x x}, u_{x x t}, u_{x t t}, u_{t t t}, \theta_{x}, \theta_{t}, \theta_{x x}, \theta_{x t}, \theta_{t t}, \\
q, q_{x}, q_{t}, q_{x x}, q_{x t}, q_{t t} \in L^{2}\left([0, \infty) ; L^{2}(\mathscr{B})\right), \\
u, u_{x}, u_{t}, u_{x x}, u_{x t}, u_{t t}, \theta-\bar{\theta}, \theta_{x}, \theta_{t}, q, q_{x}, q_{t} \rightarrow 0 \\
\text { uniformly on } \mathscr{B}, \text { as } t \rightarrow \infty, \\
u_{x x}, u_{x t}, u_{t t}, \theta_{x}, \theta_{t}, q, q_{x}, q_{t} \rightarrow 0 \text { in } L^{2}(\mathscr{B}), \text { as } t \rightarrow \infty,
\end{gathered}
$$

and

$$
u_{x}(x, t)>-1, \quad \theta(x, t)<0 \quad \forall x \in \mathscr{B}, t \geq 0 .
$$

REMARKs. 1. It follows from Eqs. (3.9), (3.10), (3.16), (3.17) and standard embedding theorems that for either the boundary-value problem or the Cauchy problem

$$
u \in C^{2}(\mathscr{B} \times[0, \infty)), \quad \theta, q \in C^{1}(\mathscr{B} \times[0, \infty)) .
$$

2. The theorems can be modified to accommodate a nonzero body force $b$ and nonzero heat supply $r$ provided $b$ and $r$ are smooth, integrable, and sufficiently small in the Sobolev sense. We must also require some boundary compatibility of $b$ and $r$ in Theorem 2 .

3. In Theorem 2 , any bounded interval can be mapped to $[0,1]$ by scaling. The choice of boundary conditions ensures that all boundary terms in the energy estimates will vanish. It may be possible to establish a similar existence theorem under other boundary assumptions, but such an undertaking is not within the scope of this paper.

4. In Theorem 2, it is not necessary to assume the thermodynamic relations. We can replace Eq. (3.5) by

$$
\begin{gathered}
\left(\frac{\hat{\sigma}-\hat{e}_{\varepsilon}}{\hat{\sigma}_{\theta}}\right)(0, \bar{\theta}, 0)>0, \\
\hat{\sigma}_{q}(0, \bar{\theta}, 0)=\hat{e}_{q}(0, \bar{\theta}, 0)=0, \\
\hat{\sigma}(\varepsilon, \theta, q)=\hat{\sigma}(\varepsilon, \theta,-q) \quad \forall q, \\
\hat{e}(\varepsilon, \theta, q)=\hat{e}(\varepsilon, \theta,-q) \quad \forall q .
\end{gathered}
$$

5. In the proof given in Sec. 4 , the order and method of estimations have been chosen sothat the proof will remain valid for both theorems. 
4. Proof of theorems. We begin the proof of Theorem 1 by choosing $\bar{\theta} \in(0, \infty)$ a fixed reference temperature. We assume that $\psi^{0}, \tau, \kappa$ belong to $C^{4}((-1, \infty) \times$ $(0, \infty))$ and that Eqs. (3.5), (3.6) hold.

The proof consists of establishing a priori estimates that allow a local solution to be continued for all time. The appropriate local existence result is recorded below in the form of a lemma. Due to the local nature of Eq. (3.6), we restrict the quantity $\left(u_{x}, \theta, q\right)$ to have values in a neighborhood of $(0, \bar{\theta}, 0)$. Thus, we choose $\delta>0$ such that

$$
\delta<\min \left(\frac{\bar{\theta}}{2}, 1\right)
$$

and such that $\hat{\sigma}_{\varepsilon},\left|\hat{\sigma}_{\theta}\right|, \hat{e}_{\theta},\left(\hat{\sigma}-\hat{e}_{\varepsilon}\right) / \hat{\sigma}_{\theta}, \tau$, and $\kappa$ are positive and bounded away from zero on

$$
\Omega=(-\delta, \delta) \times(\bar{\theta}-\delta, \bar{\theta}+\delta) \times(-\delta, \delta) .
$$

(For convenience, we will say that the domain of both $\tau$ and $\kappa$ is $\Omega$ and the third argument always has the value zero.) Let us denote

$$
\begin{gathered}
m_{1}=\inf _{\Omega} \hat{\sigma}_{\varepsilon}>0, \quad m_{2}=\inf _{\Omega}\left|\hat{\sigma}_{\theta}\right|>0, \quad m_{3}=\inf _{\Omega} \hat{e}_{\theta}>0, \\
m_{4}=\inf _{\Omega} \frac{\hat{\sigma}-\hat{e}_{\varepsilon}}{\hat{\sigma}_{\theta}}>0, \quad m_{5}=\inf _{\Omega} \tau>0 .
\end{gathered}
$$

LEMma. Suppose the assumptions of Theorem 1 hold and let $u_{0}, u_{1}, \theta_{0}, q_{0}: \mathscr{B} \rightarrow \mathbb{R}$ be given with

$$
\begin{gathered}
u_{0}^{\prime}, u_{1}, \theta_{0}, q_{0} \in H^{2}(\mathscr{B}), \\
\left(u_{0}^{\prime}(x), \theta_{0}(x), q_{0}(x)\right) \in \Omega \quad \forall x \in \mathbb{R} .
\end{gathered}
$$

Then the initial-value problem (3.1)-(3.4) has a unique solution $(u, \theta, q)$ on a maximal time internal $\left[0, T_{0}\right)$, for some $T_{0}>0$, with

$$
\begin{aligned}
& u_{x}, u_{t}, u_{x x}, u_{x t}, u_{t t}, u_{x x x}, u_{x x t}, u_{x t t}, u_{t t t}, \theta-\bar{\theta}, \theta_{t}, \\
& \quad \theta_{x}, \theta_{t t}, \theta_{x t}, \theta_{x x}, q, q_{x}, q_{t}, q_{x x}, q_{x t}, q_{t t} \\
& \quad \in C\left(\left[0, T_{0}\right) ; L^{2}(\mathscr{B})\right)
\end{aligned}
$$

and

$$
\left(u_{x}(x, t), \theta(x, t), q(x, t)\right) \in \Omega \quad \forall x \in \mathscr{B}, t \in\left[0, T_{0}\right) .
$$

Moreover, if

$$
\begin{gathered}
\sup _{t \in\left[0, T_{0}\right)} \int_{\mathscr{B}}\left(u_{x}^{2}+u_{t}^{2}+u_{x x}^{2}+u_{x t}^{2}+u_{t t}^{2}+u_{x x x}^{2}+u_{x x t}^{2}+u_{x t t}^{2}+u_{t t t}^{2}\right. \\
+(\theta-\bar{\theta})^{2}+\theta_{x}^{2}+\theta_{t}^{2}+\theta_{x x}^{2}+\theta_{x t}^{2}+\theta_{t t}^{2} \\
\left.+q^{2}+q_{x}^{2}+q_{t}^{2}+q_{x x}^{2}+q_{x t}^{2}+q_{t t}^{2}\right)(x, t) d x<\infty
\end{gathered}
$$

and

$$
\sup _{\mathscr{B} \times\left[0, T_{0}\right)}\left|u_{x}\right|<\delta, \quad \sup _{B \times\left[0, T_{0}\right)}|\theta-\bar{\theta}|<\delta, \quad \sup _{B \times\left[0, T_{0}\right)}|q|<\delta,
$$

then $T_{0}=x$. 
The result stated in the lemma follows directly from the results of Hughes, Kato, and Marsden [13]. Details of how to apply [13] can be found in Sec. 5.

We now wish to show that if Eq. (3.7) is satisfied with $\rho$ sufficiently small then Eqs. (4.8) and (4.9) hold for the solution $(u, \theta, q)$ given by the lemma. To facilitate the argument, we set

$$
\begin{aligned}
& U_{0}=\left\|u_{0}^{\prime}\right\|_{H^{2}}^{2}+\left\|u_{1}\right\|_{H^{2}}^{2}+\left\|\theta_{0}-\bar{\theta}\right\|_{H^{2}}^{2}+\left\|q_{0}\right\|_{H^{2}}^{2}, \\
& E(t)=\sup _{s \in[0, t)} \int_{\mathscr{B}}\left(u_{x}^{2}+u_{t}^{2}+u_{x x}^{2}+u_{x t}^{2}+u_{t t}^{2}+u_{x x x}^{2}+u_{x x t}^{2}+u_{x t t}^{2}+u_{t t t}^{2}\right. \\
& +(\theta-\bar{\theta})^{2}+\theta_{x}^{2}+\theta_{t}^{2}+\theta_{x x}^{2}+\theta_{x t}^{2}+\theta_{t t}^{2} \\
& \left.+q^{2}+q_{x}^{2}+q_{t}^{2}+q_{x x}^{2}+q_{x t}^{2}+q_{t t}^{2}\right)(x, s) d x \\
& +\int_{0}^{t} \int_{\mathscr{B}}\left(u_{x x}^{2}+u_{x t}^{2}+u_{t t}^{2}+u_{x x x}^{2}+u_{x x t}^{2}+u_{x t t}^{2}+u_{t t t}^{2}\right. \\
& +\theta_{x}^{2}+\theta_{t}^{2}+\theta_{x x}^{2}+\theta_{x t}^{2}+\theta_{t t}^{2}+q^{2}+q_{x}^{2} \\
& \left.+q_{t}^{2}+q_{x x}^{2}+q_{x t}^{2}+q_{t t}^{2}\right)(x, s) d x d s \quad \forall t \in\left[0, T_{0}\right), \\
& \nu(t)=\sup _{\substack{x \in \mathscr{B} \\
s \in[0, t)}}\left(u_{x x}^{2}+u_{x t}^{2}+\theta_{x}^{2}+\theta_{t}^{2}+q_{x}^{2}+q_{t}^{2}\right)^{1 / 2}(x, s) \quad \forall t \in\left[0, T_{0}\right) \text {, } \\
& \mu(t)=\nu(t)+\nu(t)^{2}+\nu(t)^{4}+\nu(t)^{8} \quad \forall t \in\left[0, T_{0}\right) .
\end{aligned}
$$

We proceed to show that for $U_{0}$ small

$$
E(t) \leq \frac{1}{4} \delta^{2} \quad \forall t \in\left[0, T_{0}\right) .
$$

It is a direct result of the Sobolev embedding theorem that

$$
\left(u_{x}^{2}+(\theta-\bar{\theta})^{2}+q^{2}\right) \leq E(t) \quad \forall x \in \mathscr{B}, t \in\left[0, T_{0}\right)
$$

from which we conclude that if Eq. (4.14) holds, both Eqs. (4.8) and (4.9) are satisfied, and hence $T_{0}=\infty$.

To establish Eq. (4.14), we derive a series of energy estimates. Let us introduce

$$
\begin{aligned}
& A(x, t)=\left[\hat{\sigma}_{\theta}^{-1}\left(\hat{\sigma}-\hat{e}_{\varepsilon}\right)\right](\varepsilon(x, t), \theta(x, t), q(x, t)), \\
& B(x, t)=A(x, t) \hat{\sigma}_{\varepsilon}(\varepsilon(x, t), \theta(x, t), q(x, t)), \\
& C(x, t)=\left[\hat{\sigma}-\hat{e}_{\varepsilon}\right](\varepsilon(x, t), \theta(x, t), q(x, t)), \\
& D(x, t)=A(x, t) \hat{\sigma}_{q}(\varepsilon(x, t), \theta(x, t), q(x, t)), \\
& E(x, t)=\hat{e}_{\theta}(\varepsilon(x, t), \theta(x, t), q(x, t)), \\
& F(x, t)=\left[\tau^{-1} \kappa \hat{e}_{q}\right](\varepsilon(x, t), \theta(x, t), q(x, t)), \\
& G(x, t)=\left[\tau^{-1} \hat{e}_{q}\right](\varepsilon(x, t), \theta(x, t), q(x, t)), \\
& H(x, t)=\left[\kappa^{-1} \tau\right](\varepsilon(x, t), \theta(x, t), q(x, t)), \\
& J(x, t)=\kappa^{-1}(\varepsilon(x, t), \theta(x, t), q(x, t)),
\end{aligned}
$$


$\forall x \in \mathscr{B}, t \in[0, \infty)$. Multiplying Eq. (3.1) by $A$ and Eq. (3.3) by $J$ yields

$$
\begin{gathered}
A u_{t t}=B u_{x x}+C \theta_{x}+D q_{x}, \\
E \theta_{t}=C u_{x t}-q_{x}+F \theta_{x}+G q,
\end{gathered}
$$

and

$$
H q_{t}+J q=-\theta_{x} \text {. }
$$

At this time we observe that differentiation of Eqs. (4.17) -(4.19) with respect to $x$ yields

$$
\begin{gathered}
A u_{x t t}=B u_{x x x}+C \theta_{x x}-A_{x} u_{t t}+B_{x} u_{x x}+C_{x} \theta_{x}+\left(D q_{x}\right)_{x}, \\
E \theta_{x t}=C u_{x x t}-q_{x x}-E_{x} \theta_{t}+C_{x} u_{x t}+\left(F \theta_{x}\right)_{x}+(G q)_{x}, \\
H q_{x t}+J q_{x}=-\theta_{x x}-H_{x} q_{t}-J_{x} q,
\end{gathered}
$$

while differentiation with respect to $t$ yields

$$
\begin{gathered}
A u_{t t t}=B u_{x x t}+C \theta_{x t}-A_{t} u_{t t}+B_{t} u_{x x}+C_{t} \theta_{x}+\left(D q_{x}\right)_{t}, \\
E \theta_{t t}=C u_{x t t}-q_{x t}-E_{t} \theta_{t}+C_{t} u_{x t}+\left(F \theta_{x}\right)_{t}+(G q)_{t}, \\
H q_{t t}+J q_{t}=-\theta_{x t}-H_{t} q_{t}-J_{t} q .
\end{gathered}
$$

The overall strategy of the proof is to obtain the inequality (4.52) below. The bulk of the proof consists of standard estimating techniques: using energy type identities and using the equations of motion to obtain estimates in terms of previously bounded quantities. Readers familiar with such techniques may wish to concentrate attention upon the derivation of Eq. (4.41). This estimate is derived from an energy identity, but does not arise in the usual fashion; the identity is motivated by the form of the inequality (2.16) and is derived from the thermodynamic relations. It is interesting to note that for the case of a bounded interval, Eq. (4.41) would follow from higher order estimates and judicious use of Poincare type inequalities; whereas in the case of an unbounded interval, the use of the thermodynamic relations is crucial to obtain the estimate.

In the following series of estimates, we will employ the elementary inequalities

$$
|a b| \leq \gamma a^{2}+\frac{1}{4 \gamma} b^{2} \quad \forall \gamma>0
$$

with the special case

$$
|a b| \leq \frac{1}{2}\left(a^{2}+b^{2}\right)
$$

and

$$
\left(\sum_{1}^{n} a_{i}\right)^{2} \leq n \sum_{1}^{n} a_{i}^{2} .
$$

We also let $\Gamma$ denote a (possibly large) generic positive constant that is independent of $T_{0}, u_{0}, u_{1}, \theta_{0}$, and $q_{0}$. We note that $\Gamma$ is allowed to depend on $\bar{\theta}, m_{1}$ through $m_{5}$, and on bounds for $\tau, \kappa, \hat{\sigma}, \hat{e}$, and their derivatives on the compact set $\bar{\Omega} \subset$ 
$((-1, \infty) \times(0, \infty) \times(-\infty, \infty))$. The following bounds are consequences of Eqs. (4.3) and (4.16):

$$
\begin{array}{llr}
A(x, t) \geq m_{4}, & B(x, t) \geq m_{1} m_{4}, & |C(x, t)| \geq m_{2} m_{4}, \\
E(x, t) \geq m_{3}, & H(x, t) \geq m_{5} M^{-1}, & J(x, t) \geq M^{-1},
\end{array}
$$

where $M$ is an upper bound for $\kappa$ on $\bar{\Omega}$.

We proceed with the basic energy estimates by multiplying Eqs. (4.20) $-(4.22)$ by $u_{x t}, \theta_{x}, q_{x}$, respectively, adding the resulting equations, and integrating over $\mathscr{B} \times[0, t)$. After some integrations by parts, we achieve the identity

$$
\begin{aligned}
\frac{1}{2} \int_{\mathscr{B}}( & \left.A u_{x t}^{2}+B u_{x x}^{2}+E \theta_{x}^{2}+H q_{x}^{2}\right)(x, t) d x+\int_{0}^{t} \int_{\mathscr{B}} J q_{x}^{2}(x, s) d x d s \\
= & \frac{1}{2} \int_{\mathscr{B}}\left(A u_{x t}^{2}+B u_{x x}^{2}+E \theta_{x}^{2}+H q_{x}^{2}\right)(x, 0) d x \\
& +\int_{0}^{t} \int_{\mathscr{B}} \frac{1}{2}\left(A_{t} u_{x t}^{2}+B_{t} u_{x x}^{2}+E_{t} \theta_{x}^{2}+H_{t} q_{x}^{2}\right)(x, s) d x d s \\
& +\int_{0}^{t} \int_{\mathscr{B}}\left(-A_{x} u_{t t}+C_{x} \theta_{x}+\left(D q_{x}\right)_{x}\right) u_{x t}(x, s) d x d s \\
& +\int_{0}^{t} \int_{\mathscr{B}}\left(-E_{x} \theta_{t}+\left(F \theta_{x}\right)_{x}+(G q)_{x}\right) \theta_{x}(x, s) d x d s \\
& +\int_{0}^{t} \int_{\mathscr{B}}\left(-H_{x} q_{t}-J_{x} q\right) q_{x}(x, s) d x d s .
\end{aligned}
$$

The relations (4.29) ensure that the left-hand side of Eq. (4.30) is bounded below by

$$
\begin{aligned}
\int_{\mathscr{B}} & \frac{1}{2}\left(m_{4} u_{x t}^{2}+m_{1} m_{4} u_{x x}^{2}+m_{3} \theta_{x}^{2}+m_{5} M^{-1} q_{x}^{2}\right)(x, t) d x \\
& +\int_{0}^{t} \int_{\mathscr{B}} M^{-1} q_{x}^{2}(x, s) d x d s .
\end{aligned}
$$

To show how to majorize the right-hand side of Eq. (4.30), we consider the following example:

$$
\begin{aligned}
\left|\int_{0}^{t} \int_{\mathscr{B}} C_{x} \theta_{x} u_{x t}(x, s) d x d s\right| & \\
= & \left|\int_{0}^{t} \int_{\mathscr{B}}\left(C_{\varepsilon} \varepsilon_{x}+C_{\theta} \theta_{x}+C_{q} q_{x}\right) \cdot \theta_{x} u_{x t}(x, s) d x d s\right| \\
\leq & \sup _{\Omega}\left(\left|C_{\varepsilon}\right|+\left|C_{\theta}\right|+\left|C_{q}\right|\right) \cdot \sup _{\substack{x \in B \\
s \in[0, t)}}\left(\left|\varepsilon_{x}\right|+\left|\theta_{x}\right|+\left|q_{x}\right|\right) \\
& \quad \int_{0}^{t} \int_{\mathscr{B}} \frac{1}{2}\left(\theta_{x}^{2}+u_{x t}^{2}\right) \leq \Gamma \nu(t) E(t) \quad \forall t \in[0, t)
\end{aligned}
$$

where we have used Eq. (4.27). All of the terms on the right-hand side of Eq. (4.30) can be majorized in a manner similar to the above example; thus we obtain the a 
priori bound

$$
\begin{aligned}
\int_{\mathscr{B}}\left(u_{x x}^{2}+u_{x t}^{2}+\theta_{x}^{2}+q_{x}^{2}\right)(x, t) d x+\int_{0}^{t} \int_{\mathscr{B}} q_{x}^{2}(x, s) d x d s \\
\leq \Gamma U_{0}+\Gamma \mu(t) E(t) .
\end{aligned}
$$

Similarly, we multiply Eqs. (4.23)-(4.25) by $u_{t t}, \theta_{t}, q_{t}$, respectively, and proceed as above to find that

$$
\begin{gathered}
\int_{\mathscr{B}}\left(u_{x t}^{2}+u_{t t}^{2}+\theta_{t}^{2}+q_{t}^{2}\right)(x, t) d x+\int_{0}^{t} \int_{\mathscr{B}} q_{t}^{2}(x, s) d x d s \\
\leq \Gamma U_{0}+\Gamma \mu(t) E(t) \quad \forall t \in[0, t) .
\end{gathered}
$$

In Eq. (4.32) we have used Eqs. (4.17)-(4.19) to write the initial values of $u_{t t}, \theta_{t}$, and $q_{t}$ as terms that appear in $U_{0}$.

To obtain energy estimates on higher order terms, we formally differentiate Eqs. (4.20)-(4.25) with respect to $t$, multiply by appropriate quantities, and integrate over $\mathscr{B} \times[0, t)$ to infer that

$$
\begin{aligned}
\int_{\mathscr{B}}( & \left.u_{x x t}^{2}+u_{x t t}^{2}+u_{t t t}^{2}+\theta_{x t}^{2}+\theta_{t t}^{2}+q_{x t}^{2}+q_{t t}^{2}\right)(x, t) d x \\
& +\int_{0}^{t} \int_{\mathscr{B}}\left(q_{x t}^{2}+q_{t t}^{2}\right)(x, s) d x d s \leq \Gamma U_{0}+\Gamma \mu(t) E(t) \quad \forall t \in[0, T) .
\end{aligned}
$$

The formal calculations can be made rigorous by employing standard techniques involving difference operators and passing to the limit.

As previously noted, we use a more unconventional technique to achieve similar bounds on the lower order terms $\left(u_{x}, u_{t}, \theta-\bar{\theta}, q\right)$. It follows from Eq. (2.17) with $r \equiv 0$ that

$$
(\theta-\bar{\theta}) \eta_{t}=-(\theta-\bar{\theta})\left(\frac{q_{x}}{\theta}+\frac{\tau q q_{t}}{\kappa \theta^{2}}\right) .
$$

Using Cattaneo's law (2.9), (2.15), (4.34) and the equation of momentum balance (2.1) with $b \equiv 0$, we conclude that

$$
\begin{gathered}
\frac{\partial}{\partial t}\left(\psi-\tilde{\psi}-\tilde{\sigma} \varepsilon+(\theta-\bar{\theta}) \eta+\frac{1}{2} u_{t}^{2}\right)+\frac{\bar{\theta}}{\kappa \theta^{2}} q^{2} \\
=\frac{\partial}{\partial x}\left((\sigma-\tilde{\sigma}) u_{t}-(\theta-\bar{\theta}) \frac{q}{\theta}\right)
\end{gathered}
$$

where $\tilde{\sigma}=\hat{\sigma}(0, \bar{\theta}, 0)$ is the residual stress and $\tilde{\psi}=\hat{\psi}(0, \bar{\theta}, 0)$. Integrating Eq. (4.35) over $\mathscr{B} \times[0, t)$ produces the result

$$
\begin{gathered}
\int_{\mathscr{B}}\left(\Lambda(\varepsilon, \theta, q)+\frac{1}{2} u_{t}^{2}\right)(x, t) d x+\int_{0}^{t} \int_{\mathscr{B}} \frac{\bar{\theta}}{\kappa(\varepsilon, \theta) \theta^{2}} q^{2}(x, s) d x d s \\
=\int_{\mathscr{B}}\left(\Lambda\left(u_{0}^{\prime}, \theta_{0}, q_{0}\right)+\frac{1}{2} u_{1}^{2}\right)(x) d x \quad \forall t \in[0, T)
\end{gathered}
$$

where $\Lambda(\varepsilon, \theta, q):=\hat{\psi}(\varepsilon, \theta, q)-\tilde{\psi}-\tilde{\sigma} \varepsilon-(\theta-\bar{\theta}) \hat{\psi}_{\theta}(\varepsilon, \theta, q)$. It follows from Eqs. (4.1), (4.3), and Taylor's theorem that

$$
\Lambda(a, b, c)+\hat{\psi}(0, \bar{\theta}, 0)-\hat{\psi}(a, \bar{\theta}, c)+\hat{\psi}_{\varepsilon}(0, \bar{\theta}, 0) a \geq \frac{1}{2} m_{3}(b-\bar{\theta})^{2} .
$$




$$
\begin{gathered}
\hat{\psi}(a, \bar{\theta}, c)-\hat{\psi}(a, \bar{\theta}, 0) \geq m_{5}(3 \bar{\theta} M)^{-1} c^{2}, \\
\hat{\psi}(a, \bar{\theta}, 0)-\hat{\psi}(0, \bar{\theta}, 0)-\hat{\psi}_{\varepsilon}(0, \bar{\theta}, 0) a \geq \frac{1}{2} m_{1} a^{2},
\end{gathered}
$$

$\forall(a, b, c) \in S$, where $M$ again is an upper bound for $\kappa$ on $\bar{\Omega}$. Adding the inequalities (4.37), (4.38), and (4.39), we produce the inequality

$$
\Lambda\left(u_{x}, \theta, q\right) \geq \frac{1}{2} m_{1} u_{x}^{2}+\frac{1}{2} m_{3}(\theta-\bar{\theta})^{2}+m_{5}(3 \bar{\theta} M)^{-1} q^{2} .
$$

Thus, we have the a priori bound

$$
\begin{aligned}
& \int_{\mathscr{B}}\left(u_{x}^{2}+u_{t}^{2}+(\theta-\bar{\theta})^{2}+q^{2}\right)(x, t) d x+\int_{0}^{t} \int_{\mathscr{B}} q^{2}(x, s) d x d s \\
& \leq \Gamma U_{0} \quad \forall t \in[0, T) .
\end{aligned}
$$

Combining the energy estimates (4.31), (4.32), (4.33), and (4.41) yields

$$
\begin{aligned}
\int_{\mathscr{B}}\left(u_{x}^{2}\right. & +u_{t}^{2}+u_{x x}^{2}+u_{x t}^{2}+u_{t t}^{2}+u_{x x t}^{2}+u_{x t t}^{2}+u_{t t t}^{2} \\
& \left.+(\theta-\theta)^{2}+\theta_{x}^{2}+\theta_{t}^{2}+\theta_{x t}^{2}+\theta_{t t}^{2}+q^{2}+q_{x}^{2}+q_{t}^{2}+q_{x t}^{2}+q_{t t}^{2}\right)(x, t) d x \\
& +\int_{0}^{t} \int_{\mathscr{B}}\left(q^{2}+q_{x}^{2}+q_{t}^{2}+q_{x t}^{2}+q_{t t}^{2}\right)(x, s) d x d s \\
\leq & \Gamma U_{0}+\Gamma \mu(t) E(t) \quad \forall t \in[0, T) .
\end{aligned}
$$

We now rewrite Eqs. (4.19), (4.22), (4.25) as

$$
\begin{gathered}
\theta_{x}=-H q_{t}-J q \\
\theta_{x x}=-\left(J q_{x}+J_{x} q+H q_{x t}+H_{x} q_{t}\right), \\
\theta_{x t}=-\left(J q_{t}+J_{t} q+H q_{t t}+H_{t} q_{t}\right)
\end{gathered}
$$

square both sides of each equation (4.43)-(4.45); integrate over $\mathscr{B}$ and $\mathscr{B} \times[0, t)$; and use Eqs. (4.28) and (4.42) to conclude that

$$
\begin{gathered}
\int_{\mathscr{B}} \theta_{x x}^{2}(x, t) d x+\int_{0}^{t} \int_{\mathscr{B}}\left(\theta_{x}^{2}+\theta_{x x}^{2}+\theta_{x t}^{2}\right)(x, s) d x d s \\
\leq \Gamma U_{0}+\Gamma \mu(t) E(t) \quad \forall t \in[0, T) .
\end{gathered}
$$

To obtain further bounds, we multiply Eq. (4.17) by $B^{-1} u_{t t}$ and Eq. (4.23) by $B^{-1} u_{t t t}$ and integrate over $\mathscr{B} \times[0, t)$. After some integrations by parts, we obtain

$$
\begin{array}{rl}
\int_{0}^{t} \int_{\mathscr{B}} & A B^{-1}\left(u_{t t}^{2}+u_{t t t}^{2}\right)(x, s) d x d s-\int_{0}^{t} \int_{\mathscr{B}}\left(u_{x t}^{2}+u_{x t t}^{2}\right)(x, s) d x d s \\
= & \int_{\mathscr{B}}\left(u_{x} u_{x t}+u_{x t} u_{x t t}\right)(x, 0) d x-\int_{\mathscr{B}}\left(u_{x} u_{x t}+u_{x t} u_{x t t}\right)(x, t) d x \\
& +\int_{0}^{t} \int_{\mathscr{B}} B^{-1} u_{t t}\left(C \theta_{x}+D q_{x}\right)(x, s) d x d s \\
& +\int_{0}^{t} \int_{\mathscr{B}} B^{-1} u_{t t t}\left(C \theta_{x t}-A_{t} u_{t t}+B_{t} u_{x x}+C_{t} \theta_{x}+\left(D q_{x}\right)_{t}\right)(x, s) d x d s .
\end{array}
$$


We then multiply Eq. (4.18) by $E^{-1} u_{x t}$ and Eq. (4.24) by $E^{-1} u_{x t t}$ and integrate over $\mathscr{B} \times[0, t)$. After some integrations by parts, we obtain

$$
\begin{aligned}
\int_{0}^{t} \int_{\mathscr{B}} C E^{-1}\left(u_{x t}^{2}+u_{x t t}^{2}\right)(x, s) d x d s \\
=\int_{\mathscr{B}}\left(u_{t} \theta_{x}+u_{t t} \theta_{x t}\right)(x, 0) d x-\int_{\mathscr{B}}\left(\left(u_{t} \theta_{x}+u_{t t} \theta_{x t}\right)(x, t) d x\right. \\
\quad+\int_{0}^{t} \int_{\mathscr{B}}\left(u_{t t} \theta_{x}+u_{t t t} \theta_{x t}\right)(x, s) d x d s \\
\quad+\int_{0}^{t} \int_{\mathscr{B}} E^{-1} u_{x t}\left(q_{x}-F \theta_{x}-G q\right)(x, s) d x d s \\
\quad+\int_{0}^{t} \int_{\mathscr{B}} E^{-1} u_{x t t}\left(q_{x t}+E_{t} \theta_{t}-C_{t} u_{x t}-\left(F \theta_{x}\right)_{t}-(G q)_{t}\right)(x, s) d x d s .
\end{aligned}
$$

It follows from adding a suitable multiple of Eq. (4.48) to Eq. (4.47) and Eqs. (4.42), (4.46) that

$$
\begin{gathered}
\int_{0}^{t} \int_{\mathscr{B}}\left(u_{t t}^{2}+u_{x t}^{2}+u_{t t t}^{2}+u_{x t t}^{2}\right)(x, s) d x d s \\
\leq \Gamma \int_{0}^{t} \int_{\mathscr{B}}\left(\left|u_{t t} \theta_{x}\right|+\left|u_{t t t} \theta_{x t}\right|\right)(x, s) d x d s \\
\quad+\Gamma U_{0}+\Gamma \mu(t) E(t) \quad \forall t \in[0, T) .
\end{gathered}
$$

Thus, using Eq. (4.26) with an appropriate choice of $\gamma$, we achieve the additional bounds

$$
\begin{gathered}
\int_{0}^{t} \int_{\mathscr{B}}\left(u_{t t}^{2}+u_{x t}^{2}+u_{t t t}^{2}+u_{x t t}^{2}\right)(x, s) d x d s \\
\leq \Gamma \mu(t) E(t) \quad \forall t \in[0, T) .
\end{gathered}
$$

It follows from squaring the equations (4.17)-(4.25), integrating over $\mathscr{B} \times[0, t]$, and appealing to Eqs. (4.42), (4.46), and (4.50) that

$$
\begin{aligned}
\int_{\mathscr{B}}\left(u_{x x x}^{2}+q_{x x}^{2}\right)(x, t) d x \\
\quad+\int_{0}^{t} \int_{\mathscr{B}}\left(u_{x x}^{2}+u_{x x x}^{2}+u_{x x t}^{2}+\theta_{t}^{2}+\theta_{t t}^{2}+q_{x x}^{2}\right)(x, s) d x d s \\
\leq \Gamma U_{0}+\Gamma \mu(t) E(t) \quad \forall t \in[0, T) .
\end{aligned}
$$

Combining Eqs. (4.42), (4.46), (4.50), (4.51) and taking the supremum over $[0, t]$ of both sides of the resulting inequality yields

$$
E(t) \leq \bar{\Gamma} U_{0}+\bar{\Gamma} \mu(t) E(t) \quad \forall t \in[0, T)
$$

where $\bar{\Gamma}$ is independent of $T, u_{0}, u_{1}, \theta_{0}$, and $q_{0}$. We choose $\alpha>0$ such that

$$
\alpha \leq \frac{1}{2} \delta^{2}, \quad \bar{\Gamma}\left(\alpha^{1 / 2}+\alpha+\alpha^{2}+\alpha^{4}\right) \leq \frac{1}{4},
$$

and then choose $\rho>0$ such that

$$
\bar{\Gamma} \rho^{2} \leq \frac{1}{4} \alpha
$$


and

$$
U_{0} \leq \rho^{2} \Rightarrow E(0) \leq \frac{1}{2} \alpha .
$$

The $\delta$ used in Eq. (4.53) is the same number introduced in Eqs. (4.1), (4.2). Suppose now that Eq. (3.7) holds with the above choice of $\rho$ so that $\bar{\Gamma} U_{0} \leq \frac{1}{4} \alpha$. Moreover, it follows from the Sobolev embedding theorem that

$$
\nu(t)^{2} \leq E(t) \quad \forall t \in[0, T),
$$

and so

$$
\mu(t) \leq\left[E^{1 / 2}+E+E^{2}+E^{4}\right](t) \quad \forall t \in[0, T) .
$$

It then follows from Eq. (4.52) that for any $t \in[0, T)$, if $E(t) \leq \alpha$ then in fact $E(t) \leq \frac{1}{2} \alpha$. Therefore, by continuity and Eq. (4.55), we have

$$
E(t) \leq \frac{1}{2} \alpha \leq \frac{1}{4} \delta^{2} \quad \forall t \in[0, T)
$$

since $E(0) \leq \frac{1}{2} \alpha$. The bound (4.58) and (4.15) allow us to conclude that both Eqs. (4.8) and (4.9) are satisfied, and thus $T_{0}=\infty$. Finally, we note that Eqs. (3.11)(3.13) hold by virtue of Eq. (4.58) and the definitions of $E$ and $\Omega$. (See, e.g., [8].)

5. Local existence. The local existence lemma stated in Sec. 4 is a consequence of the paper by Hughes, Kato, and Marsden [13]. To see this, we first introduce

$$
v(x, t)=u_{t}(x, t), \quad \varphi(x, t)=\theta(x, t)-\bar{\theta} .
$$

We note that Eqs. (4.17)-(4.19) can be written as

$$
\frac{\partial U}{\partial t}+\mathscr{A}(U) U=0
$$

where

$$
\mathscr{A}(W) U=\left(\begin{array}{c}
U=(\varepsilon, v, \varphi, q)^{\mathrm{T}}, \\
-v_{x} \\
-A^{-1}(W)\left[B(W) \varepsilon_{x}+C(W) \varphi_{x}+D(W) q_{x}\right] \\
-E^{-1}(W)\left[C(W) v_{x}-q_{x}+F(W) \varphi_{x}+G(W) q\right] \\
H^{-1}(W)\left[\varphi_{x}+J(W) q\right]
\end{array}\right),
$$

and $A, B$, etc. are motivated by Eq. (4.16). We take the spaces $X, Y, Z$, and $Z^{\prime}$ of the paper to be

$$
Y=\left[H^{2}(\mathbb{R})\right]^{4}, \quad X=\left[H^{1}(\mathbb{R})\right]^{4}, \quad Z=Z^{\prime}=\left[H^{0}(\mathbb{R})\right]^{4} .
$$

Let $W \in Y$ and introduce the operators $N(W)$, a norm on $Z$, such that

$$
\begin{array}{r}
\|U\|_{N(\boldsymbol{W})}^{2}=\int_{\mathbb{R}}\left(B(W) \varepsilon^{2}+A(W) v^{2}+E(W) \varphi^{2}+2\left[D E C^{-1}\right](W) q \varphi\right. \\
\left.+\left[H+H D F C^{-1}\right]\left(W^{\prime}\right) q^{2}\right)
\end{array}
$$

and

$$
S(W) \equiv S=I-\Delta=I-\frac{\partial^{2}}{\partial x^{2}}
$$


where $I$ is the identity operator on $Y$. The norm $N(W)$ is equivalent to the usual norm on $Z$ by virtue of the boundedness of the coefficients on the set $\Omega$ and the lower bounds expressed in Eq. (4.29). The operator $S$ is an isomorphism from $Y$ to $Z$ and $\mathscr{A}$ generates a quasi-contractive $C_{0}$-semigroup. The assumptions of Theorem I of [13] are then satisfied, and the local existence lemma stated in Sec. 4 follows.

For the case of a bounded interval, we can adapt the theorem of the paper by modifying the spaces $X, Y, Z$, and $Z^{\prime}$. Denote the space of $H_{\mathrm{loc}}^{m}$ functions that are periodic with period 2 by $H_{p}^{m}$ and equip this space with the norm

$$
\|u\|_{m, p(\mathbb{R})}=\|u\|_{m}(-1,1)
$$

where $\|\cdot\|_{m}(-1,1)$ is the usual norm on $H^{m}(-1,1)$. Then replace the spaces in Eq. (5.2) with their periodic analogs and replace the norm Eq. (5.3) with its analog. For prescribed periodic initial data, we are guaranteed a periodic solution $(\varepsilon, v, \varphi, q)$. We extend the initial data (defined originally on $[0,1]$ ) to $\mathbb{R}$ so that $u_{0}^{\prime}, \theta_{0}$ are even periodic functions of $x$ and $u_{1}, q_{0}$ are odd periodic functions of $x$. The compatibility conditions (3.15) ensure that the extended initial data will lie in the space $Y$. Consider the set $\mathscr{V}$ of vector-valued functions with first and third components even and second and fourth components odd. We previously imposed a symmetry condition (3.21) on the stress and internal energy functions of the form

$$
\hat{\sigma}(\varepsilon, \theta, q)=\hat{\sigma}(\varepsilon, \theta,-q), \quad \hat{e}(\varepsilon, \theta, q)=\hat{e}(\varepsilon, \theta,-q) .
$$

The structure of $\mathscr{A}$ indicates that the solution operator of Eq. (5.1) is invariant on $\mathscr{V}$. Therefore, the solution of Eq. (5.1) has the property that $\varepsilon, \theta$ are even periodic functions and $v, q$ are odd periodic functions so that the boundary conditions (3.14) are automatically satisfied.

Acknowledgment. The author wishes to thank the referee for his helpful suggestions.

\section{REFERENCES}

[1] C. Cattaneo, Sulla coduzione del calore, Atti Sem. Mat. Fis. Univ. Modena 3, 83-101 (1948)

[2] D. S. Chandrasekharaiah, Thermoelasticity with second sound: a review, Appl. Mech. Rev. 39, 355-376 (1986)

[3] P. J. Chen and M. E. Gurtin, On second sound in materials with memory, Z. Angew. Math. Phys. 21, 232-241 (1970)

[4] B. D. Coleman, M. Fabrizio, and D. R. Owen, On the thermodynamics of second sound in dielectric solids, Arch. Rat. Mech. Anal. 80, 135-158 (1982)

[5] B. D. Coleman, M. Fabrizio, and D. R. Owen, Il secundo suono nei cristalli: termodinamica ed equazioni costitutive, Rend. Sem. Mat. Univ. Padova 68, 208-277 (1982)

[6] B. D. Coleman and V. J. Mizel, Thermodynamics and departures from Fourier's law of heat conduction, Arch. Rat. Mech. Anal. 13, 245-261 (1963)

[7] B. D. Coleman and W. Noll, The thermodynamics of elastic materials with heat conduction and viscosity, Arch. Rat. Mech. Anal. 13, 167-178 (1963)

[8] B. D. Coleman, W. J. Hrusa, and D. R. Owen, Stability of equilibrium for a nonlinear hyperbolic system describing heat propagation by second sound in solids, Arch. Rat. Mech. Anal. 94, 267-289 (1986)

[9] W. A. Day, The Thermodynamics of Simple Materials with Fading Memory, Springer-Verlag, Berlin, 1970 
[10] N. Fox, Generalized thermoelasticity, Internat. J. Engrg. Sci. 7, 437-445 (1968)

[11] M. E. Gurtin and A. C. Pipkin, A general theory of heat conduction with finite wave speeds, Arch. Rat. Mech. Anal. 31, 113-126 (1968)

[12] W. J. Hrusa and M. A. Tarabek, On smooth solutions of the Cauchy problem in one-dimensional nonlinear thermoelasticity, Quart. Appl. Math. 47, 631-644 (1989)

[13] T. J. R. Hughes, T. Kato, and J. E. Marsden, Well-posed quasi-linear second-order hyperbolic systems with applications to nonlinear elastodynamics and general relativity, Arch. Rat. Mech. Anal. 63, 273-294 (1977)

[14] D. D. Joseph and L. Preziosi, Heat waves, Rev. Modern Phys. 61, 41-73 (1989)

[15] P. D. Lax, Development of singularities of solutions of nonlinear hyperbolic partial differential equations, J. Math. Phys. 5, 611-613 (1964)

[16] R. C. MacCamy and V. J. Mizel, Existence and non-existence in the large of solutions of quasilinear wave equations, Arch. Rat. Mech. Anal. 25, 299-320 (1967)

[17] I. Müller, The coldness, a universal function in thermoelastic bodies, Arch. Rat. Mech. Anal. 4, 319-332 (1971) 